\title{
RESISTENCIA A IRSE SOLO A LA CAMA: ESTUDIO DE CASO
}

\author{
FRANCISCO J. MOLINA \\ Universidad de Almería \\ (Recibido el 12 de marzo de 1998)
}

\begin{abstract}
Se presenta un informe referido a la intervención llevada a cabo en un problema de sueño con un niño de dos años. El análisis funcional del problema resalta la relevancia de la actuación de los padres ante el comportamiento problemático del niño. Desde ahí se planifica una manera de prevenir sencilla y poco intrusiva que muestra ser tremendamente efectiva después de una semana, y garante de la adherencia al tratamiento por parte de los adultos, con quienes se trabaja directamente, moldeando las pautas a llevar a cabo más que instruyéndoles.
\end{abstract}

Palabras clave: Problemas de sueño, análisis funcional, adherencia, no intrusividad.

Bedtime tantrums: A case study

It is presented a report concerned with an intervention in a sleep problem with a two year-old child. The functional analysis of the problem emphasizes the importance of the parent's behavior in relation to the child's problematic behavior. The work is made directly with the parents shaping instead of instructing the guide lines of the treatment. This natural intervention with minimal intrusitivity was very effective after a week, and increased the likehood of the adult's adherence behavior.

Key words: Sleep problems, functional analysis, adherence, no intrusitivity.

\section{INTRODUCCIÓN}

Un motivo habitual de preocupación para los padres tiene que ver con el comportamiento de dormir de sus hijos. Desde el nacimiento hasta que un niño se va a dormir sin que nadie se lo tenga que indicar, la instauración del hábito de dormir se prolonga a través de ciertos momentos críticos que exigen a los padres ir (re)ajustando horas y lugares: (a) desde que el bebé despierte varias veces durante la noche para comer, hasta dormir de un tirón, (b) de dormir en la cuna, a hacerlo en la cama, c) de compartir habitación con sus padres, a hacerlo solo. En cómo se lleven a cabo estos cambios (reacciones del niño y reaccio-

Agradecimientos: Quiero agradecer a la Dra. M. Carmen Luciano la revisión del manuscrito inicial y sus comentarios al respecto. Estos han servido de guía para la configuración final del escrito que se presenta. nes de los padres a las de éste) puede encontrarse la génesis de algún problema (Molina, 1996).

Entre los más frecuentes en la infancia se documentan como problemas de sueño las negativas a irse a dormir o quedarse solo en la cama, levantarse durante la noche e ir hasta la habitación de los padres (lo que Brockmann (1990) refiere como el visitante nocturno), despertarse demasiado temprano, despertarse atemorizado, etc.

A continuación se describe cómo se desarrolló el análisis y planificó la modificación de un patrón de sueño inadecuado en un niño de 2 años de edad. El planteamiento de intervención, a través del análisis y cambio del comportamiento de

Correspondencia: Departamento de Ciencias de la Salud y Psicología Clínica. Facultad de Humanidades y Ciencias de la Educación. Universidad de Almería. La Cañada de San Urbano. 04120 Almería. Tf́no.: 950215 205. Fax: 950215 203. Correo-e: igomez@ ualm.es. 
los padres, se alinea en una manera de hacer que pretende ser sencilla a la vez que eficaz y garante de la adherencia de los adultos a las pautas prescritas por el especialista (Luciano y Herruzo, 1992a; Molina, Gómez, Luciano y Herruzo, 1993).

\section{PRESENTACIÓN DEL CASO}

Pablo es un niño de 2 años. Su desarrollo sigue un cauce de normalidad en todas las áreas de interés.

En lo que tiene que ver con el sueño, aspecto de relevancia en este contexto, cabe destacar que a los seis meses de su nacimiento los padres de Pablo consiguieron instaurar en el niño un patrón de sueño valorado como plenamente satisfactorio. Hasta ese momento, recuerdan algún episodio aislado referido a alguna mala noche en la que el niño presentó un sueño intranquilo (despertarse varias veces, llanto, etc.) debido a la acumulación de gases: lo que podría denominarse, de acuerdo con la literatura especializada, cólico del infante (por ejemplo, Haslam, 1985).

Desde los seis meses, los padres de Pablo establecieron una rutina previa al sueño nocturno que se mantuvo, con ligeras variaciones hasta la aparición del problema que será descrito más adelante. El patrón de sueño del niño en el último año se caracterizaba, grosso modo, por la secuencia que determina el baño, cena, unos minutos de interacción relajada y, aún despierto, a la cuna (10 de la noche, aproximadamente). Pablo permanecía en la cuna en silencio hasta conciliar el sueño, que se prolongaba, sin interrupción, durante las siguientes 10 horas. Pasado ese tiempo sus padres lo levantaban ( $\sin$ que, en muchos casos, llegara a despertarse siquiera levemente) para que tomara un biberón ( 8 de la mañana, aproximadamente). Después, el niño seguía durmiendo, en la cuna, durante dos horas más; hasta que se despertaba y jugueteaba en la cuna, balbuceaba y, más tarde, llamaba a papá o a mamá. El patrón de sueño se completaba con una siesta de 90 minutos tras el almuerzo.

Pablo dormía en su propia habitación desde los siete meses.

\section{EVOLUCIÓN Y CARACTERIZACIÓN DEL PROBLEMA}

A los dos años, los padres de Pablo comenzaron a observar que algo empezaba a ir mal cuando llegaba el momento de que su hijo se fuera a dormir, hecho éste que coincidió con el final del período vacacional y el cambio de la cuna a la cama (desplazamientos frecuentes, cambios en rutinas y horarios que obligaron en ocasiones, incluso, a que Pablo durmiera en la misma cama con sus padres). Los padres describen una situación en la que el período previo a irse a la cama se prolongaba cada vez un poco más (Pablo traía otro cuento que quería que le leyeran, otro álbum de fotos que repasar, etc., con el beneplácito de sus padres). Si le decían al niño que iban a acostarlo éste protestaba y acercaba otro cuento. Cuando, finalmente, lo llevaban hasta la cama Pablo se negaba a quedarse en ella solo: lloraba, decía "mamá/papá aquí» (señalando la cama), se agarraba al cuello o a la ropa del adulto y, si los padres salían de la habitación dejándole acostado, Pablo se levantaba e iba tras sus pasos. Finalmente el niño se quedaba dormido si alguno de sus padres permanecía en la habitación con él. Esta situación parece que varió con rapidez desde estar de pie en la habitación mientras el niño (en la cama) conciliaba el sueño, hasta estar tumbados en la cama con él, pasando por estar sentados en el suelo (junto a la cama) y sentados en su cama (todo ello, en la mayoría de las ocasiones manteniendo contacto físico con el niño).

Así, en el momento en que los padres de Pablo coinciden en que hay un pro- 
blema al que buscar solución, su hijo únicamente permanece en su cama hasta quedar dormido si está acompañado por alguno de ellos. Si no es así, el niño se levanta y acude al lugar en donde estos están: el sofá, su propia cama, etc.

\section{ANÁLISIS E INTERVENCIÓN}

La intervención se fundamentó en el análisis funcional de las respuestas de dormir de Pablo y en la actuación de sus padres. En palabras de Ayllón (1992), «la causa de los problemas de la persona va a ser encontrada en cómo ella está interactuando con su ambiente social (por lo que la terapia) consistirá primariamente en alterar las condiciones responsables de la forma en que las personas se relacionan unas con otras" (p. 352). De acuerdo con esto, el análisis de la conducta de Pablo pasa necesariamente por la consideración de la conducta de sus padres.

Estos, ante las variaciones del comportamiento del niño inducidas por los cambios generados en sus rutinas diarias, comenzaron a actuar (a relacionar su comportamiento con el del niño) de manera que se fueron seleccionando determinadas maneras de responder a Pablo (aquéllas que traían a sus padres junto a él). Cambiaron la rutina del patrón de inicio del sueño. Más adelante, cuando la nueva interacción estuvo consolidada, se presentó con suficiente claridad como para que los padres del niño coincidieran en que tenían un problema y un objetivo claro: Pablo debería quedarse en la cama sin protestar, retomando el patrón previo a la aparición del problema.

Así, tras el baño y la cena se establecen las condiciones para que los momentos previos a irse a la cama sean tranquilos: sentados en el sofá se lee un cuento, se ven fotos, etc. Cuando llega la hora (establecida por los padres) de que Pablo se vaya a dormir la consigna es ignorar las quejas del niño, que son más probables a partir de este punto. Es decir, los padres de Pablo no deben decir y/o hacer nada en relación con las quejas de su hijo. Se conducirá, pues, al niño hasta su cuarto y se procederá como antes: un beso, despedida de buenas noches, apagar la luz, dejarlo en la cama, y salir de la habitación. En este punto se establecen las siguientes consideraciones, de suma importancia:

(a) Pablo puede comenzar a llorar. Los padres deberán abstenerse de acudir hasta donde se encuentra el niño. Y esto por más que llore (o grite) su hijo.

(b) El niño puede levantarse de la cama e ir tras los pasos de sus padres. Estos deberán coger al niño con suavidad y, sin decir nada y manteniendo la calma, volver a depositarlo en la cama mientras le dicen «debes acostarte a dormir" (o algo similar a lo que le dicen cuando, por primera vez, lo acuestan y se despiden hasta el día siguiente). Esto deberán hacerlo tantas veces como se levante Pablo después de dejarlo acostado y siempre de la misma forma, atendiendo a no introducir variaciones de una vez a otra.

Los padres deciden que sea el padre el que inicie el tratamiento y que, después de unos días, se implique también la madre.

\section{RESULTADOS}

Pablo lloraba y salía tras su padre cuando éste le dejaba en su cama. Ignorando tales llantos, Pablo fue devuelto una y otra vez a su cama hasta que permanecía en ella llorando pero sin levantarse. Como era de esperar, las primeras noches el llanto del niño se incrementó en intensidad y varió en topografía (gritos, súplicas para que su papá permaneciera con él, negativas explícitas: "no quiero", 
demandas explícitas: «ahí (señalando hacia fuera de la habitación)", etc.).

Como se puede apreciar en la Figura 1, el número de veces que Pablo hubo de ser devuelto a su cama fue decreciendo paulatinamente. El séptimo día después de haber puesto en práctica la estrategia de cambio, Pablo permaneció en su cama sin levantarse y sin llorar cuando su padre lo depositó en ella por primera vez en la noche. Después de una semana «sin problema" la madre del niño lo llevó a dormir sin que apareciera ninguna demanda especial de atención por parte de su hijo. Desde ahí, en las semanas siguientes, los padres del niño informan del mantenimiento de los resultados y de que, incluso, alguna noche Pablo ha pedido irse a dormir.

Tres meses después de iniciada la intervención los resultados alcanzados se mantenían. Pablo permanecía despierto, y sin protestar, en la cama una vez que su padre o su madre lo acostaban, y seguía en ella hasta quedarse dormido una vez que abandonaban su habitación.

\section{DISCUSIÓN}

El abordaje terapéutico que acaba de ser presentado se caracterizaría por su sencillez y eficacia, lo que hace que esta intervención psicológica pueda ser calificada como tremendamente parsimoniosa. Desde el principio se huye de explicaciones más o menos tautológicas (descriptivas más que explicativas), de la apelación a traumas de naturaleza ciertamente difusa como presunta causa psicológica (cuando en verdad sólo son descripciones / nominaciones técnicas, aunque no por ello menos redundantes, del propio hecho objeto de análisis).

Esta visión no "psicopatologizante» del problema, focalizada en la historia de las interacciones del comportamiento del niño y sus padres, tiene como primer efecto una mejor comprensión de parte de los adultos implicados y, desde ahí, la intervención con mínimos elementos y haciendo uso de las habilidades que los padres del niño ya tienen. Es decir, otra vez se busca el ajuste al repertorio com-

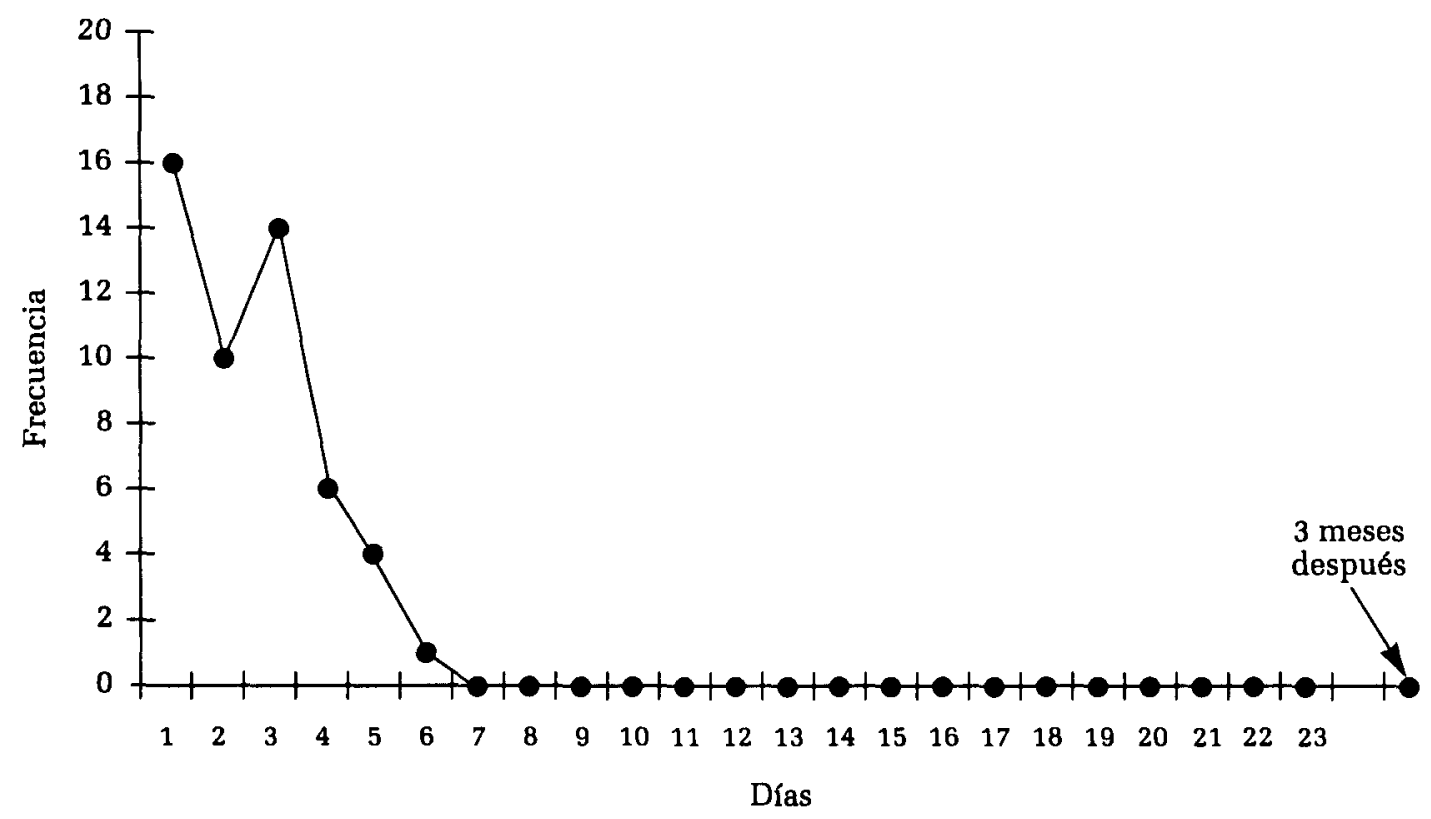

Figura 1. Ocasiones en que Pablo fue llevado de vuelta a su cama tras ser acostado 
portamental de quienes, en última instancia, serán los encargados de producir los cambios necesarios que definirán la intervención terapéutica, lo que facilitará la adherencia a las prescripciones (Luciano y Herruzo, 1992b). El objetivo se plantea en términos de recuperar el patrón de sueño que Pablo ya tuvo. La manera de alcanzarlo es que sus padres se vuelvan a comportar como entonces en relación a él.

Por otra parte, desde el análisis de la situación se podía formular un pronóstico favorable: la historia del problema no se extendía mucho, existía un patrón de reforzamiento continuo, y las variables que delimitaban la situación problema estaban claramente determinadas (Luciano, 1996).

Cabe señalar que la manipulación planificada de variables (técnicamente, extinción) suele tener unos efectos indeseables suficientemente documentados (por ejemplo, Cruzado y Labrador, 1997; Gelfand y Hartmann, 1989; Ross, 1991; Sulzer-Azaroff y Mayer, 1991) como para que, ajustados a cada caso, puedan (y deban) ser informados a quienes van a ponerla en práctica: en este caso, los padres de Pablo. De no ser así, al desconocer que la variabilidad comportamental (incluso emocional) es un producto normal de la intervención que se lleva a cabo, podrían llegar a pensar que algo se está haciendo mal y abandonar las pautas recién puestas en marcha, lo que además de infructuoso resultaría contraproducente.

Este efecto se vio minimizado en este caso en tanto que no sólo hubo extinción de las pautas o episodios inapropiados, sino que los comportamientos adecuados de Pablo - previos a dormir - fueron atendidos convenientemente por sus padres. De este modo, la atención que antes recibía el niño por sus comportamientos inadecuados no desapareció de la interacción de sus padres sino que tan sólo cambió la contingencia en la que ésta se proporcionaba. Es decir, se cambian las funciones del comportamiento, pero no se elimina la atención de los padres respecto de la vida diaria de Pablo (Ayllón y Simon, 1982). No obstante, la información que se proporciona a los padres sobre los efectos iniciales de la extinción, a pesar de lo anterior, les permitió elegir (ajustar) el repertorio de uno u otro a la hora de afrontar la situación. De esta manera, la probabilidad de cumplir su compromiso se vería incrementada.

Una vez superado el esfuerzo inicial de (auto)control en la situación problemática, los padres de Pablo se encuentran con que, de una manera sencilla y mínimamente costosa para ellos, han conseguido restablecer un patrón de sueño que ya conocían y consideraban el adecuado para su hijo. Y, más importante aún, han sido ellos los responsables de la mejora, con una orientación no iatrogénica (Luciano, 1995; Pérez Álvarez, 1996).

\section{REFERENCIAS BIBLIOGRÁFICAS}

Ayllón, T. (1992). Implicaciones clínicas de algunos conceptos del Conductismo Radical de Skinner. En J. Gil Roales-Nieto, M.C. Luciano y M. Pérez (Eds.), Vigencia de la obra de Skinner (pp. 351-358). Granada: Universidad de Granada.

Ayllón, T. y Simon, S. (1982). Behavior therapy with children. En J. Lanchenmeyer y M. Gibbs (Eds.), Psychopathology in childhood (pp. 272-297). New York: Gardner Press.

Brockmann, E. (1990). El visitante nocturno. En G. Aguilar, B.G. de Lebl, y L.A. Recinos (Eds.), Conductas problema en el niño normal. Programas preventivos y terapéuticos. Guía para padres y maestros (pp. 65-71). México: Trillas.

Cruzado, J.A. y Labrador, F.J. (1997). Técnicas para la reducción de conductas operantes. En F.J. Labrador, J.A. Cruzado y M. Muñoz (Eds.), Manual de técnicas de modificación 
y terapia de conducta (pp. 501-531). Madrid: Pirámide.

Gelfand, D.M. y Hartmann, D.P. (1989). Análisis y terapia de la conducta infantil. Madrid: Pirámide.

Haslam, D. (1985). Trastornos del sueño infantil. Normas prácticas de tratamiento. Barcelona: Martínez-Roca.

Luciano, M.C. (1995). Análisis y modificación de conducta en infancia y adolescencia. Proyecto docente e investigador. Universidad de Almería.

Luciano, M.C. (1996). Evaluación de las alteraciones en la infancia. En M.C. Luciano (Dir.), Manual de psicología clínica. Infancia y adolescencia (pp. 155-203). Valencia: Promolibro.

Luciano, M.C. y Herruzo, J. (1992a). Dos estudios clínicos: (1) alteraciones del lenguaje e interacción social, y (2) huida de casa y problemas afectivos. Psicothema, 5, 213-228.
Luciano, M.C. y Herruzo, J. (1992b). Some relevant components of adherence behavior. Journal of Behavior Therapy and Experimental Psychiatry, 23, 117-124.

Molina, F.J. (1996). Trastornos del sueño en la infancia. En M.C. Luciano (Dir.), Manual de psicología clínica. Infancia y adolescencia (pp. 355-386). Valencia: Promolibro.

Molina, F.J., Gómez, I., Luciano, M.C. y Herruzo, J. (1993). Conductas problema relacionadas con el dormir. Estudio de un caso. Análisis y Modificación de Conducta, 19, 433-451.

Pérez Álvarez, M. (1996). Tratamientos psicológicos. Madrid: Universitas.

Ross, A.O. (1991). Terapia de la conducta infantil. Principios, procedimientos y bases teóricas. México: Limusa.

Sulzer-Azaroff, B. y Mayer, G.R. (1991), Procedimientos del análisis conductual aplicado con niños y jóvenes. México: Trillas. 